\title{
Q1RT2008

\section{Method of automatic recognition of helicopters flying at low altitudes}

\author{
by Henryk Madura, Mirosław Dąbrowski, Tomasz Sosnowski, Piotr Trzaskawka
}

Military University of Technology, ul. Gen. Sylwestra Kaliskiego 2, 00-908 Warszawa, Poland

Key words: temperature measurement, thermography.

The use of thermal radiation for target recognition is a very effective method, since every object emits certain amounts of thermal energy that can't be fully masked. In recent years a rapid development of IR target recognition devices can be observed. These devices are capable of detection and identification of low-flying objects at close distances. Such Infrared Search and Track Systems (IRSTs) successfully replace radar-based devices in air surveillance and weapon control applications.

Effective detection of objects in infrared range can be performed when characteristic emissive parameters of objects to be detected are known. Knowledge of those parameters makes it possible to distinguish the target from other sources of thermal radiation. The set of such parameters is called the thermal signature of a target. The data required for further analysis were gathered by recording numerous thermal images of selected target in various weather conditions. The recordings were performed using thermal cameras with uncooled microbolometer focal plane arrays $(7.5 \mu \mathrm{m}$ to $13 \mu \mathrm{m}$ working range).

The multi-purpose helicopter was chosen as a selected target to be detected. It is an object capable of lowaltitude flying at various speeds. Such helicopters are commonly used in military operation and pose a serious threat to enemy forces. Their efficient detection is then an important task for air defense systems.

A helicopter as the object visualized in the infrared range can be divided into two basic areas, characterized by different emissive properties: a steel, matt-painted fuselage (emissivity coefficient $\varepsilon=0.95-0.98$ ) and windows made of organic glass (emissivity coefficient $\varepsilon=0.86)^{1}$. A thermal characteristic of a helicopter is analyzed during flight conditions, with fully warmed engines, which are the main source of thermal emission.

The background in analyzed measurement scenario can be very complex and diverse. Both natural and artificial, man-made objects can appear in the background of a thermal image. The cycle of thermal recordings made it possible to include the background characteristics in the target detection algorithm.

For the detection method, a set of parameters needs to be defined that influence the target detection. In the applied algorithm such parameters are: the mean temperature of the background, object-to-background thermal contrast and average object temperature distribution. Detection range was modeled using NVTherm software, which can estimate the parameters of a thermal device at the design stage. (Fig.1) The modeled device was equipped with $384 \times 288$ milobolometer focal plane array by ULIS (U03081).

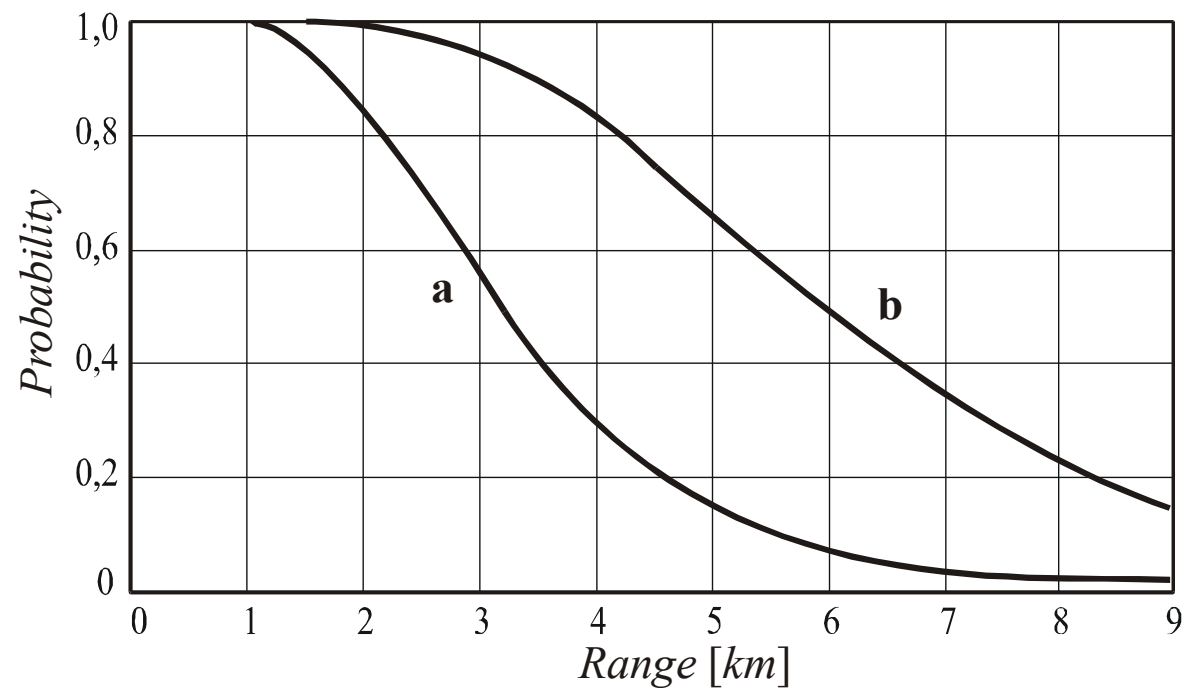

Fig. 1. Simulated probability of helicopter detection by a thermal scanner $a$ - front view, $b$ - side view.

The presented method can be used for the detection of low-flying objects in full horizontal angle at various distances from thermal scanner with 3D properties of an object taken into account.

After the device is turned on, the ambient temperature measurement is performed and based on the result the operating range is set. In the next step $k$ thermal images are recorded in order to determine the reference 
image $\left(\mathrm{O}_{\mathrm{k}}\right)$. At this stage constant elements of visualized thermal scene are eliminated. When the reference image is derived, the process of target searching may begin. During this process, the current image $O_{B}$ is recorded and then reference image is subtracted from it. As a result, the difference image is obtained, which pixel $\mathrm{R}_{\mathrm{i}, \mathrm{j}}$ values can be described as:

$$
R_{i, j}=\left|T_{i, j}\left(L_{B}\right)-T_{i, j}\left(L_{N}\right)\right|
$$

where $T_{i, j}\left(L_{B}\right)$ is the temperature at $(i, j)$ point of actual image, $T_{i, j}\left(L_{k}\right)$ is the temperature in corresponding point of reference image. Simultaneously, the reference image is updated in order to include possible background changes caused by variable atmospheric conditions.

Temperature changes detected in the difference image $\left(\mathrm{R}_{\mathrm{i}, \mathrm{j}}\right)$ are compared with detection threshold $\left(\mathrm{T}_{\text {det }}\right)$ to eliminate small temperature variations in the observed scene from further analysis. Remaining points, for which the condition $R_{i, j}>T_{\text {det }}$ is fulfilled, are processed through selected combination of digital image transformations. At this stage the random objects with thermal characteristics not matching assumed pattern are eliminated. The thermal properties of the remaining separate areas obtained as a result of digital transformations are then analyzed and recognition parameter $\mathrm{Pr}_{0}$ for each area is calculated. This parameter is then compared with boundary conditions derived from thermal measurements of real objects. If the relation $\mathrm{Pr}_{\min }<\mathrm{Pr}_{\mathrm{o}}<\mathrm{Pr}_{\max }$ is fulfilled then the it is assumed that the detection of target took place and appropriate information is transmitted.

The simulations performed by the authors lead to the conclusion that the presented method is effective and price-competitive alternative for commercially available IRST systems. Proper methods of analysis of thermal images lead to the significant reduction of false alarms, which is an important factor in assessment of the quality of IR tracking and detection systems.

The concept of detection of low-flying objects presented here can be applied in both military and civilian systems for airspace surveillance. When paired with intelligent weapon systems it may substantially improve the capabilities of air defense.

\section{REFERENCES}

[1] H. Madura, and M. Kołodziejczyk, "Influence of sun radiation on results of non-contact temperature measurements in far infrared range", Opto-Electronics Review 13 (3), 2005.

[2] M. Dabrowski, and R. Dulski, "Computer faceted thermal model of helicopter”, Proceedings of VI International Conference on Quantitative Infrared Thermography, Dubrovnik 24-27 September 2002, pp. 244-249.

[3] H. Madura, M. Dabrowski, and R. Dulski, „Simulations of radiative properties of a helicopter in IR”, 7-th International Workshop on Advanced Infrared Technology and Applications, Pisa 9-11 September 2003, pp. $42-43$ 